\title{
The Projective Synchronization of Fractional-order Multi-scroll Chaotic Systems \\ Xiaodan Shao ${ }^{1}$, Feng Chen ${ }^{1, \text { a }}$ \\ ${ }^{1}$ College of Electronics and Information Engineering, Southwest University, Chongqing, 400715, P.R. China \\ aEmail address: Fengchen.uestc@gmail.com (F. Chen)
}

\begin{abstract}
Keywords: Projective synchronization, fractional-order, chaos, multi-scroll.
Abstract. Projective synchronization is an efficient method in the study of synchronization. This paper presents the projective synchronization between a fractional-order multi-scroll chaotic system and the designed response system. By making the output synchronization error system of the studied system satisfy the stability conditions of the fractional-order chaotic system, this paper verifies that projective synchronization can be achieved between the two systems. The performance of error dynamics and the dynamical behaviors of the studied system are numerically analyzed in some detail. Simulation results demonstrate a good match between theory and experiment.
\end{abstract}

\section{Introduction}

Chaos synchronization, an important topic in nonlinear science, has gained a lot of attention in a variety of research fields over the last two decades [1,2]. Projective synchronization, one of the synchronization methods, means that the drive and response system could be synchronized up to a scaling factor and the study of it can improve our understanding of chaotic synchronization [3].

In the present paper, we consider the projective synchronization of the fractional-order multi-scroll chaotic systems, which have complicated topological structure and a rich variety of dynamical behaviors. Multi-scroll chaotic attractors have two or more scrolls and they have a rich variety of dynamical behaviors [4]. Fractional multi-scroll chaotic systems have been reported in literature [5, 6]. In Refs. [7], the author also studied the synchronization of fractional-order systems.

The rest of this paper is organized as follows. A brief description of the projective synchronization method of fractional order chaotic systems is presented in section 2. Section3 presents the projective synchronization of fractional-order multi-scroll chaotic system. Numerical simulation results are shown in section 4. Finally, some conclusions are given in section 5.

\section{Projective Synchronization Method of Fractional-Order Chaotic Systems}

We consider a fractional-order chaotic system and apply the projective synchronization method in [3], the system given by:

$$
\frac{d^{\alpha} X}{d t^{a}}=f(X(t))=\hat{A} X(t)+\hat{h}(X(t))
$$

where $X=(x 1, x 2, \ldots, x n)^{T} \in R^{n}$ is the system state variable, $f: R^{n} \rightarrow R^{n}$ is vector fields inn-dimensional space, $A X(t)$ is the linear term of $f(X(t)), \hat{h}(X(t))$ is the nonlinear term of $f(X(t))$. By discomposing $A X(t)$, we can obtain $A X(t)=A X(t)+\bar{A} X(t)$, where $A$ is full rank matrix with negative values for all the real part of the eigenvalues. Let $h(X(t))=\bar{A} X(t)+\hbar X(t)$, then (1) can be rewritten as (2) and the corresponding response system is (3).

$$
\frac{d^{\alpha} X}{d t^{a}}=A X(t)+h(X(t))
$$




$$
\frac{d^{\alpha} Y}{d t^{a}}=A Y(t)+h(X(t)) / s .
$$

where $Y(t) \in R^{n}, s$ is a given scale factor. Define the error of system (2) and system (3) as $e(t)=X(t)-s Y(t)$, then the error system can be obtained as (4). As all the real part for the eigenvalues of $A$ are negative, according to Lemma 1 , when $t \rightarrow \infty$, the error system (4) is stable about its zero equilibrium. Thus, the projective synchronization is achieved between system (2) and (3).

$$
\frac{d^{\alpha} e(t)}{d t^{a}}=\frac{d^{\alpha} X(t)}{d t^{a}}-s \frac{d^{\alpha} Y(t)}{d t^{a}}=A X(t)-s A Y(t)=A e(t)
$$

Lemma 1 For fractional-order chaotic systems as [8], if the system is linear, the system can be written as $D^{\alpha} X=A X, X \in R^{n}, A \in R^{n \times n}$, which obey the rule: for fractional order linear systems with $0<\alpha<1$, if the real part of all eigenvalues are in none-positive value, then the system is stable.

\section{The Projective Synchronization of Fractional-order Multi-scroll Chaotic System}

In this section, we apply the above same analytical method on fractional-order multi-scroll chaotic systems. Consider a novel 3 scroll chaotic attractors model [7] as the drive system:

$$
\begin{aligned}
& \frac{d^{\alpha} x}{d t^{a}}=a(x-y)-y z, \\
& \frac{d^{\alpha} y}{d t^{a}}=-b y+x z, \\
& \frac{d^{\alpha} z}{d t^{a}}=-c z+d x+x y .
\end{aligned}
$$

where $a, b, c, d \in R^{+}$are real constant parameters of this system. $x, y, z$ are the system state variables. The nonlinear term of the drive system (5) can be written as $h(t)$. The linear term of the drive system (5) is $\hat{A}$. The eigenvalues of $\hat{A}$ are $a,-b,-c$. In order to satisfy the Lemma 1 , we rewritten $\hat{A}$ as $A$. The expressions of $h(t), \hat{A}$, and $A$ are as follows:

$$
h(t)=\left[\begin{array}{c}
(a-p) x-y z \\
x z \\
x y
\end{array}\right], \quad \hat{A}=\left[\begin{array}{ccc}
a & -a & 0 \\
0 & -b & 0 \\
d & 0 & -c
\end{array}\right], \quad A=\left[\begin{array}{ccc}
p & -a & 0 \\
0 & -b & 0 \\
d & 0 & -c
\end{array}\right] .
$$

It is easy to find that when $p<0$, the real parts of the eigenvalues of $A$ are negative. The response system corresponding to (5) is (6).

$$
\begin{aligned}
& \frac{d^{\alpha} u}{d t^{a}}=a(u-v)+((a-p) x-y z) / s, \\
& \frac{d^{\alpha} v}{d t^{a}}=-b v+x z / s, \\
& \frac{d^{\alpha} w}{d t^{a}}=-c w+d u+x y / s .
\end{aligned}
$$


By making the output synchronization error system of studied systems satisfy Lemma 1, the projective synchronization between (5) and (6) can be realized in theory.

\section{Numerical simulations}

To begin with, when $\alpha=0.9$, we set $a=4.15, b=10, c=4, d=0.1$, and scaling factor $s=2$. We set $p=-8$, then the system eigenvalues of synchronization error system are all negative, thus the system stability conditions are satisfied. The error curve $e(t)$ of the projective synchronization for system (5) and system (6) is shown in Fig.1. We find that the time response of the synchronization error $e(t)$ tends to zero as $t \rightarrow \infty$, which means that the synchronization between the two systems is achieved at $s=2$. Both system (5) and system (6) generate four-scroll chaotic attractors, the trajectories of them in three-dimensional space are shown in Fig.2 (a) and 2(b) respectively. The phase plots on the $x-z$ plane are shown in Fig. 2(c) and 2(d). Fig. 2 demonstrates that the system (5) is twice the amplitude of the system (6), and they are in-phase in the end.
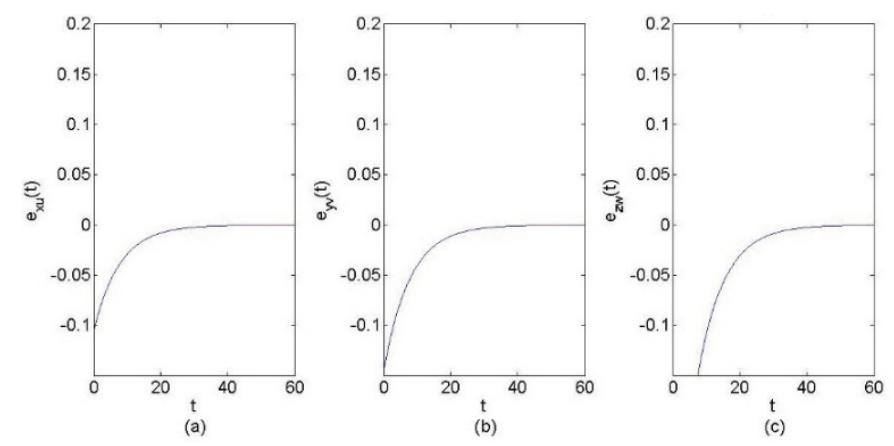

Fig. 1. The error curve of projective synchronization for system (5) and (6), $p=-8, s=2$ (a) response curve of $e_{x u}(t)$. (b) response curve of $e_{y v}(t)$ (c) response curve of $e_{z w}(t)$.
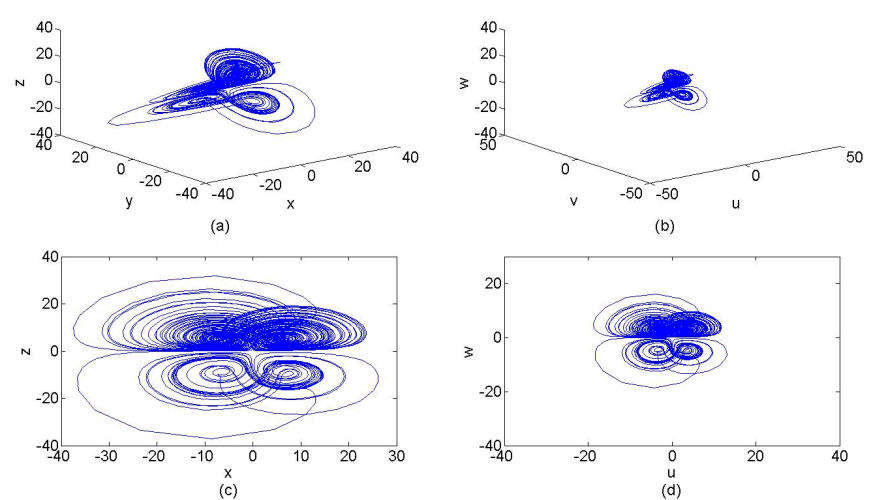

Fig. 2. The four-scroll chaotic attractors generated when system (5) and (6) reached projective synchronization, $p=-8, s=2$.
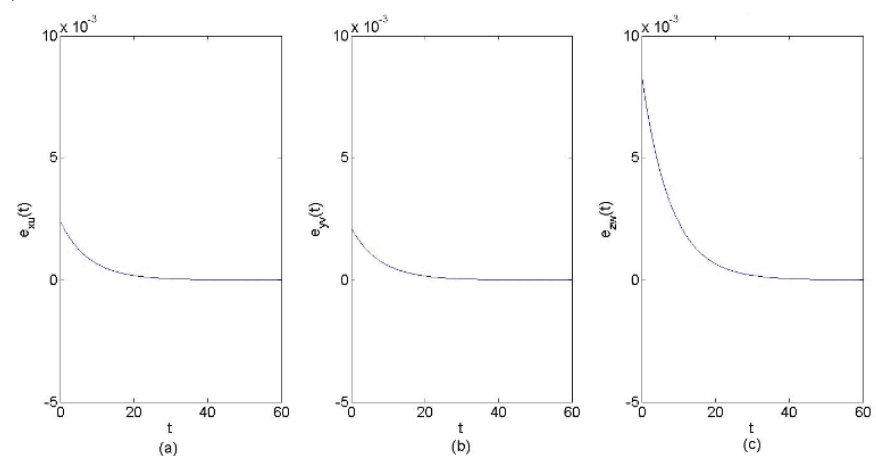

Fig. 3. The error curve of projective synchronization for system (5) and (6), $p=-8, s=-2$ (a) response curve of $e_{x u}(t)$. (b) response curve of $e_{y v}(t)$ (c) response curve of $e_{z w}(t)$. 


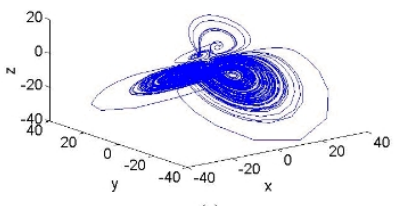

(a)
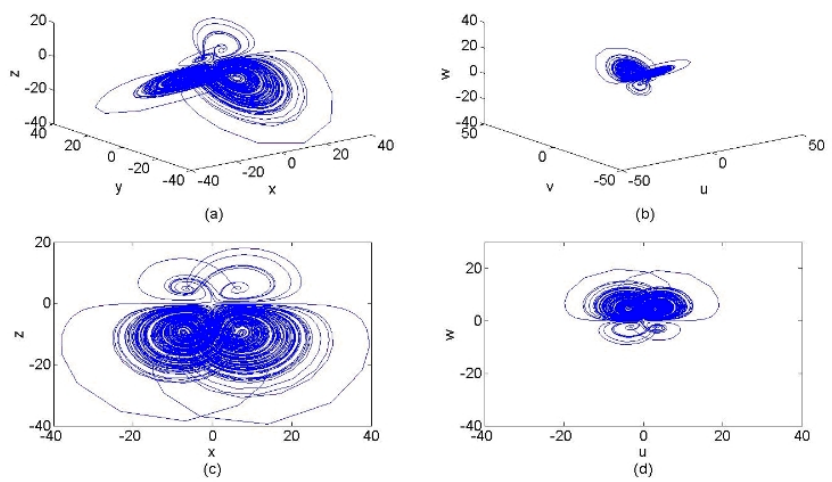

(b)

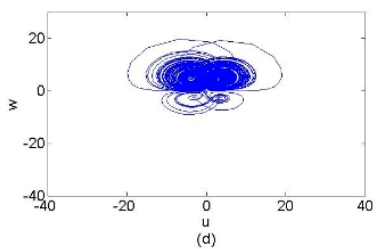

Fig. 4. The four-scroll chaotic attractors generated when system (5) and (6) reached projective synchronization, $p=-8, s=-2$.

Next, we reset the value of parameter $s$ is -2 without changing the other system parameters. From Fig. 3 and Fig.4, we know that the output of system (5) is twice the amplitude of the system (6), but they are anti-phase. The projective synchronization between the two systems is achieved at $s=-2$.

\section{Conclusions}

In this paper, we have designed the response system corresponding to a fractional-order multi-scroll chaotic system to investigate the projective synchronization. Using the fractional-order chaotic system theory, some stability conditions for output synchronization error system of studied system were carried out. The simulations have shown that the projective synchronization of two systems is achieved at its scaling factor $s=2$ and the output of fractional-order multi-scroll chaotic system was twice the amplitude of the corresponding response system, and they were in-phase. When $s=-2$, the same results can be obtained except that the phases of two systems were opposite. In the further work, we will investigate how the fractional order change effect the projective synchronization.

\section{Acknowledgements}

This work was supported in part by the Fundamental Research Funds for the Central Universities un der grant number XDJK2014C018 and Doctoral Fund of Southwest University (No.SWU113067).

\section{References}

[1] Pecora, L. M, Carroll, T. L. Phys. Rev. Lett. 1990, 64(11), 821-824.

[2] Rosenblum, M. G, Pikovsky, A. S, Kurths: Phys. Rev. Lett. 1997, 78(22), 4193-4196.

[3] Xingjie Wang, Yijie He: Acta Phys. Sin. 2008, 57(3), 1485-1492.

[4] L.Wang: Nonlinear Dyn. 2009, 56(4), 453-462.

[5] W. H. Deng, J. H. L“u. Phys. Lett. A, 369(2007), 438-443.

[6] Feng. Chen, Lei. Xia, Daqing Guo, Ying. Liu: Computational Science 10: 4 (2013) 1203-121.

[7] Chunguang Li , Xiaofeng Liao, Juebang Yu: Phys. Rev. E, 68 (2003), 067203.

[8] Matignon, D. Proceeding of IMACS, IEEE-SMC. 1996, 963-968. 